\title{
Propagation of Neuronal Activity along the Neocortical-Perirhinal- Entorhinal Pathway in the Guinea Pig
}

\author{
Gerardo Biella, Laura Uva, and Marco de Curtis \\ Department of Experimental Neurophysiology, Istituto Nazionale Neurologico, 20133 Milan, Italy
}

The study of synaptic interactions within the parahippocampal region is crucial to understand the integrative functions performed by this region during memory information processing. Despite the extensive anatomical studies, the intrinsic physiology of the parahippocampal area has been poorly investigated. We describe here the organization pattern of the synaptic network formed by the temporal neocortex, areas 36 and 35 of the perirhinal cortex (PRC) and the entorhinal cortex (EC), in the in vitro isolated guinea-pig brain. Current source density analysis of laminar field potential profiles was performed with multichannel silicon probes positioned in different parahippocampal subfields. Stimulation of the temporal neocortex induced monosynaptic and polysynaptic potentials in areas 35 and 36 , respectively. Area 36 stimulation evoked monosynaptic responses within areas 36 and 35 . Stimuli in area 35 induced responses that propagated longitudinally along area 35 itself. No local field responses were observed in the EC after stimulation of both neocortex and areas 35/36. Despite the absence of a local extracellular response, intracellular recordings demonstrated that subpopulations of superficial layer neurons in medial and lateral EC showed polysynaptic EPSPs after stimulation of area 35 and area 36.

The results demonstrate that the propagation of neuronal activity across the rhinal sulcus in the direction from the PRC to the EC is finely and diffusely distributed. In agreement with previous reports, these findings suggest that the PRC-EC pathway is highly regulated by inhibitory network interactions.

Key words: current source density; entorhinal cortex; isolated guinea pig brain preparation; parahippocampal region; perirhinal cortex; neocortex
Memory-related functions in the parahippocampal region are performed by the interactions between neurons that form its major subregions, the entorhinal cortex (EC) and the perirhinal cortex (PRC) (Zola-Morgan et al., 1989; Braak and Braak, 1993; Meunier et al., 1993; Suzuki, 1996; Brown and Xiang, 1998; Amaral, 1999; Brown and Aggleton, 2001). The associative connections within and between EC and PRC have been investigated extensively with tracing and lesion techniques (Van Hoesen and Pandya, 1975; Swanson and Köhler, 1986; Lopes da Silva et al., 1990; Burwell et al., 1995; Insausti et al., 1997; Burwell and Amaral, 1998a,b). These studies recognized two major systems that vehicle visuospatial and olfactory inputs to the hippocampus (Wilson and Steward, 1978; Otto et al., 1991; Eichenbaum and Otto, 1993; Naber et al., 1997; Biella and de Curtis, 2000). The neocortical afferents from unimodal and polymodal cortical areas project to the PRC (Suzuki and Amaral, 1994; Burwell et al., 1995; Suzuki, 1996), from which activity is supposedly transmitted to the hippocampus proper (Steward, 1976; Lopes da Silva et al., 1990; Witter, 1993) either directly (Naber et al., 1997) or via a pathway mediated by the EC (Burwell and Amaral, 1998a). The anatomical data, therefore, suggest the existence of a sequential cascade of synaptic interactions that transfer the information

\footnotetext{
Received June 28, 2002; revised Aug. 14, 2002; accepted Sept. 3, 2002.

The study was sponsored by the European Community Project VSAMUEL (Grant IST 99-1-1-A) and by the Italian Health Ministry. Multichannel silicon probes were kindly provided by the University of Michigan Center for Neural Communication Technology, sponsored by National Institutes of Health National Center for Research Resources Grant P41-RR09754. We thank Solange van der Linden of the Institute of Neurobiology of the University of Amsterdam for participating in preliminary experiments.

Correspondence should be addressed to Marco de Curtis, Department of Experimental Neurophysiology, Istituto Nazionale Neurologico, via Celoria 11, 20133 Milan, Italy. E-mail: decurtis@istituto-besta.it.

Copyright () 2002 Society for Neuroscience 0270-6474/02/229972-08\$15.00/0
}

generated in the neocortex (NC) to the hippocampus via the PRC and the EC. In addition, within both the PRC and the EC, intrinsic fiber systems form an associative network predominantly developed along a rostrocaudal dimension (Witter et al., 1986; Burwell and Amaral, 1998a; Dolorfo and Amaral, 1998).

Despite the number of anatomical studies, the electrophysiological correlate of the associative connectivity between the PRC and the EC have received little attention because of the difficulties to simultaneously access the rhinal region with stimulating and recording electrodes in the in vivo condition. On the other hand, in vitro slices do not allow the preservation of sufficient synaptic circuitry to achieve a complete analysis of the interactions between EC and PRC. Previous attempts to study this pathway in the fluoroethane-anesthetized cat in vivo (G. Pelletier and D. Paré, unpublished observations) and in rodents in vitro (M. P. Witter, G. Biella, and T. Iijima, unpublished observations) demonstrated that PRC stimulation determined no detectable responses in the EC, suggesting that such a pathway is under a powerful inhibitory control. It has been demonstrated recently that the circuit activated within area 36 by stimulation of the neocortex is characterized by a pronounced, possibly feedforward, inhibition (Biella et al., 2001; Martina et al., 2001) that prevents the firing of neurons of the superficial layers and controls the propagation of the activity across the rhinal sulcus to the subsequent synaptic relay. In the present study, we analyze the electrophysiological patterns of propagation from the neocortex to the EC by analyzing the associative cortico-cortical interactions between the rhinal cortices of the guinea pig. The study has been primarily facilitated by the use of the in vitro isolated brain preparation, which allows for precise positioning of stimulating and recording electrodes in cortical areas readily identified by direct visual inspection. 
Parts of this work have been published previously in abstract form (Biella et al., 2000).

\section{MATERIALS AND METHODS}

After barbiturate anesthesia (sodium pentothal, $20 \mathrm{mg} / \mathrm{kg}$, i.p.), brains of young adult guinea pigs (150-200 gm weight) were dissected out according to the standard procedure described in detail previously (de Curtis et al., 1991, 1998; Muhlethaler et al., 1993). The isolated brain was arterially perfused at $5.5 \mathrm{ml} / \mathrm{min}$ with a solution containing the following: $126 \mathrm{~mm}$ $\mathrm{NaCl}, 3 \mathrm{~mm} \mathrm{KCl}, 1.2 \mathrm{~mm} \mathrm{KH}_{2} \mathrm{PO}_{4}, 1.3 \mathrm{~mm} \mathrm{MgSO}_{4}, 2.4 \mathrm{~mm} \mathrm{CaCl}_{2}, 26 \mathrm{~mm}$ $\mathrm{NaHCO}_{3}, 15 \mathrm{~mm}$ glucose, $2.1 \mathrm{~mm}$ HEPES, and 3\% dextran (molecular weight, 70.000), oxygenated with a $95 \% \mathrm{O}_{2}-5 \% \mathrm{CO}_{2}$ gas mixture, $\mathrm{pH} 7.3$. Experiments were performed at $32^{\circ} \mathrm{C}$. Bipolar stimuli were delivered via either custom-made twisted silver wires or tungsten electrodes arrays formed by two or three electrode pairs (200-400 $\mu \mathrm{m}$ separation), each vertically separated by $500-1000 \mu \mathrm{m}$ (Frederick Haer Co., Bowdoinham, $\mathrm{ME}$ ) positioned at different depths within cortical structures. Current stimuli of 50-150 $\mu \mathrm{A}$ and $200-500 \mu \mathrm{sec}$ were used. Extracellular recordings were performed with either glass micropipettes (filled with $1 \mathrm{M}$ $\mathrm{NaCl}$ ) or stainless steel electrodes. Laminar depth profiles were performed with silicon probes that featured 16 recording sites separated by $50 \mu \mathrm{m}$ on a single shaft [kindly provided by Jamille Hetke (Center of Neural Communication and Technology, University of Michigan, Ann Arbor, MI)]. The position of the electrodes and the probes could be easily and rapidly modified during the experiment under direct visual control with a stereoscopic microscope. The field potentials were amplified [Biomedical Engineering (Thornwood, NY) extracellular amplifier], were digitized via an ATMIO-64E3 National Instruments (Milan, Italy) board, and were stored on tape (Bio-Logic, Claix, France) for off-line analysis. Intracellular recordings were performed with micropipettes filled with $2 \mathrm{M}$ potassium acetate and $2 \%$ biocytine. The intracellular signals were recorded with a Neurodata Instruments (New York, NY) amplifier. Data acquisition and analysis were performed by using a Labview software (developed in our department by G. Biella). Current source density analysis (CSD) was performed with a $200 \mu \mathrm{m}$ differentiation grid on laminar profiles recorded with the 16-channel silicon probes according to the standard procedure (Ketchum and Haberly, 1993; Biella and de Curtis, 1995, 2000).

Electrolytic lesions performed at the end of the experiments were used to mark the position of both the stimulating electrodes and the silicon probes (Biella and de Curtis, 2000, their Methods). When intracellular biocytine injections were performed, brains were processed for biocytine-HRP visualization. After fixation in $4 \%$ paraformaldehyde, $100 \mu \mathrm{m}$ sections were cut by a vibratome. Intracellular biocytine was revealed with $\mathrm{ABC}$ kit (Vector Laboratories, Burlingame, CA) on sections counterstained with thionin.

\section{RESULTS}

Experiments were performed on 43 isolated guinea pig brains. Figure $1 A$ shows the $\mathrm{NC}$, the PRC, and the EC in guinea pig brain coronal sections (Uva et al., 2001), as identified on the basis of cytoarchitectonic criteria described previously in the rat (Insausti et al., 1997; Burwell and Amaral, 1998a). The PRC can be distinguished from the NC for a broad layer II and a less distinct subdivision in six layers. In area 35, layer II neurons are more sparse, and layer IV is thinner compared with area 36 . The presence of a lamina dissecans marks the border between area 35 and the EC. The EC was subdivided in a lateral and medial areas (1-EC and m-EC, respectively) (Biella and de Curtis, 2000; Biella et al., 2002). The study focuses on the EC and the PRC; no recordings were performed in the most caudal part of the rhinal region, named postrhinal cortex.

The general pattern of propagation of excitation from the NC to the PRC and the EC was investigated in six experiments by positioning recording electrodes in areas 36 and 35 and at different sites in the medial and in the lateral bands of the EC parallel to the rhinal sulcus, as schematically illustrated in the left panel of Figure $1 B$. Stimuli applied to the superficial layers of the parietaltemporal NC region (3-4 mm dorsal to area 36) evoked a surfacepositive-depth-negative early potential, followed by a surface- negative-depth-positive potential in area 36, close to the stimulation site (Fig. 1B, left column; recordings performed at 600 $\mu \mathrm{m}$ depth). NC stimulation induced a late potential in area 35 (Fig. $1 B$, second trace in the left column) and a low-amplitude response in the EC (Fig. $1 B$, bottom traces in the left column). Stimulation of deep NC layers induced no responses in both PRC and EC (data not shown; $n=6$ ). Stimulation of superficial layers in area 36 determined an early response within area 36 and in area 35 (Fig. 1B, middle column) and a delayed potential in the lateral EC. No clear local response was induced within area 36 by local stimulation of deep layers (data not shown; $n=4$ ). Finally, stimulation of area 35 induced a large response within area 35 and a late potential in the l-EC and in the m-EC.

To evaluate the pattern of local network activation in different cortical areas, extracellular currents distribution was evaluated with CSD analysis performed on field potential laminar profiles recorded with 16-channel silicon probes separated by $50 \mu \mathrm{m}$ (Biella and de Curtis, 2000). Figure $2 A$ describes typical responses recorded in area 36 after stimulation of the temporal $\mathrm{NC}$ and area 36 (Fig. 2A, scheme in the inset and histological control in the left panel) $(n=9)$ (Biella et al., 2001). The early potential components of the NC- and area 36-evoked responses correlated with an early sink that peaked at $\sim 10 \mathrm{msec}$ latency, located at 250-400 $\mu \mathrm{m}$ depth and coupled to a superficial current source (Fig. 2A, compare delays of the responses in the potentials recorded at different depths to those in the CSD profile). Such events were followed by a large current dipole that peaked at 15-20 msec, in coincidence with the surface-positive response (Fig. 2A). We demonstrated recently that, in area 36, the early and late sinks correspond to a monosynaptic EPSP and a disynaptic EPSP superimposed to a presumably feedforward IPSP (Biella et al., 2001).

To study the activity propagation pattern from area 36 to area 35 and within area 35 itself, 16-channel probe recordings were performed in area 35. A sequence of early and late sink-source current dipoles similar to that described in area 36 was observed after stimulation in area 36 (Fig. $2 B$, right panel) $(n=7)$. The early sink was more widely distributed (between 200 and $400 \mu \mathrm{m}$ depth) because of the thicker layer I typical of area 35. Surfacepositive potentials correlated to late CSD sinks at a delay longer than 15-20 msec were observed in response to NC stimulation in superficial layers (Fig. $2 B$, middle panel) $(n=4)$. The position of the recording silicon probe in area 35 (arrowhead) and the stimulating electrodes in the NC (arrow) are illustrated in the left panel in Figure $2 B$. In three experiments, the propagation of activity within area 35 was evaluated by performing recordings close to and between 1.5 and $2 \mathrm{~mm}$ away from the stimulation electrode in area 35 itself (Fig. $3 A$ ). At the recording site close to the stimulating electrode, a small-amplitude sequence of early-late sinks with the typical spatial distribution described for the are 36-evoked responses was observed (Fig. 3B, left panel). A late potential associated with a superficial sink at $0-200 \mu \mathrm{m}$ depth was consistently observed at sites remote to the stimulating electrode (Fig. 3B, right panel). The amplitude of current sinks and sources observed in area 35 was much smaller than those in area 36, possibly because of the cytoarchitectonic features of this region (see Discussion).

Recordings with 16-channel silicon probes were performed in the EC after stimulation in the $\mathrm{NC}$ and in areas 36 and 35 of the PRC (Fig. 4A). To identify the borders between the l-EC and the $\mathrm{m}-\mathrm{EC}$, the responses to lateral olfactory tract (LOT) stimulation were used (Fig. 4B) (Biella and de Curtis, 2000). LOT-evoked potentials in the m-EC (bottom laminar profile) typically showed 

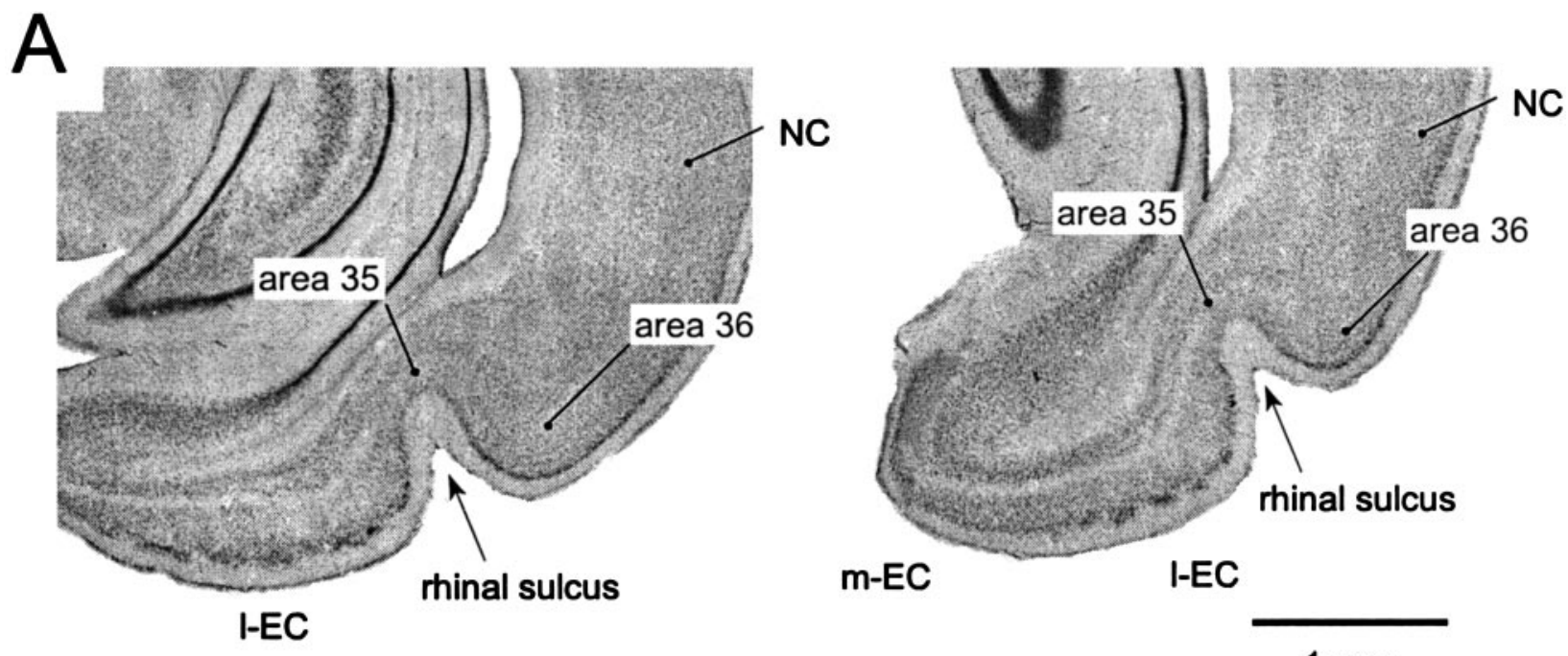

$1 \mathrm{~mm}$

B

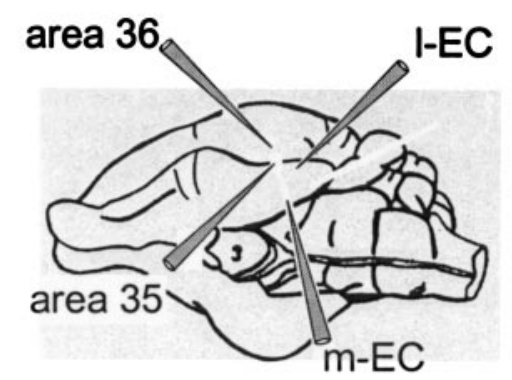

st. NC

st. 36

st. 35
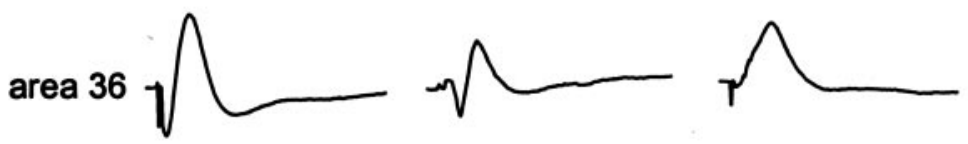

area 35
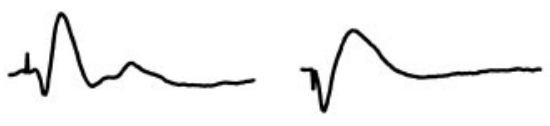

I-EC
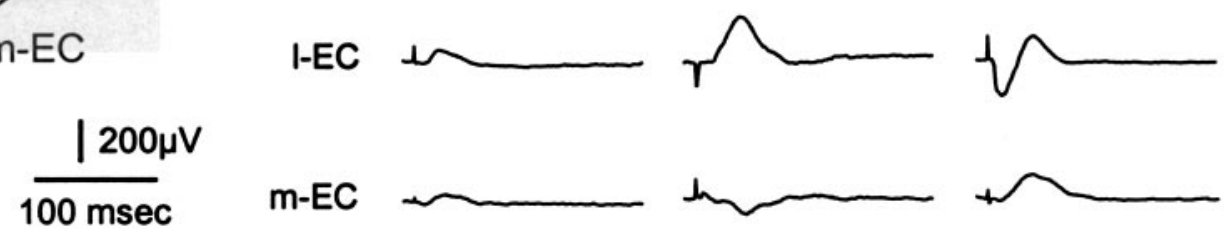

m-EC
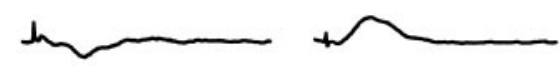

Figure 1. A, Coronal sections of the rhinal region of the guinea pig at two different rostrocaudal levels. The NC, area 36, and area 35 in the perirhinal cortex, the m-EC, and the 1-EC are indicated. The rhinal sulcus is indicated by the arrowhead. B, Field responses evoked in area 36, area 35, 1-EC, and $\mathrm{m}$-EC by surface stimulation of the NC (left column), area 36 (middle column), and area 35 (right column). A schematic drawing of the positions of the recording electrodes is shown on the left. All recordings were performed at 500-600 $\mu \mathrm{m}$ depth.

a longer delay than 1-EC responses (top laminar profile). Profiles recorded in $\mathrm{m}-\mathrm{EC}$ and $\mathrm{l}-\mathrm{EC}$ after stimulation in $\mathrm{NC}$, in area 36 , and in area 35 did not show local reversal of activity (Fig. 4C). The absence of a locally generated field response was also confirmed by the absence of local current sinks-sources during CSD analysis of laminar profiles (data not shown). Therefore, the observed potentials are not generated within the EC but represent volume-conducted far field responses. Recordings of field potential profiles at different caudodorsal and lateromedial sites in both 1-EC and m-EC confirmed the absence of locally generated EC responses. These finding contrast with the results of tracing studies that show a well defined pattern of connectivity between the PRC and the EC (Van Hoesen and Pandya, 1975; Burwell and Amaral, 1998a).

Taking in consideration that the absence of a detectable EC field response could be attributable to the sparse distribution of the afferent fibers on a small number of EC neurons scattered in space (Biella et al., 2001, their Discussion), we studied further the interactions between NC-PRC and the EC by performing intra- cellular recordings in principal EC neurons of superficial layers (layers II and III), which are known to receive the largest input from the PRC and mediate the propagation of information to the hippocampus. Intracellular recordings were performed on 82 layer II-III EC neurons that showed an average membrane potential of $-67.26 \pm 7.16 \mathrm{mV}$ (mean $\pm \mathrm{SD}$ ). Neurons in 1-EC typically showed EPSPs with a shorter delay from LOT stimulation than m-EC cells. At resting membrane potential, none of the neurons generated an action potential in response to both LOT and PRC stimulation. Fifteen neurons were morphologically identified after intracellular biocytine injection as stellate and pyramidal cells (see Materials and Methods). In seven experiments, intracellular recordings in layer II-III EC neurons were performed simultaneously to extracellular laminar profiles with a 16-channel silicon probe during area 36 stimulation. In the representative experiment illustrated in Figure 4D, area 36 stimulation induced a late depolarizing potential (Fig. 4D, top trace) in a cell identified as a stellate neuron (as shown in Fig. 4E). A simultaneous field potential was recorded with a 16-channel probe at a nearby position 


\section{A}

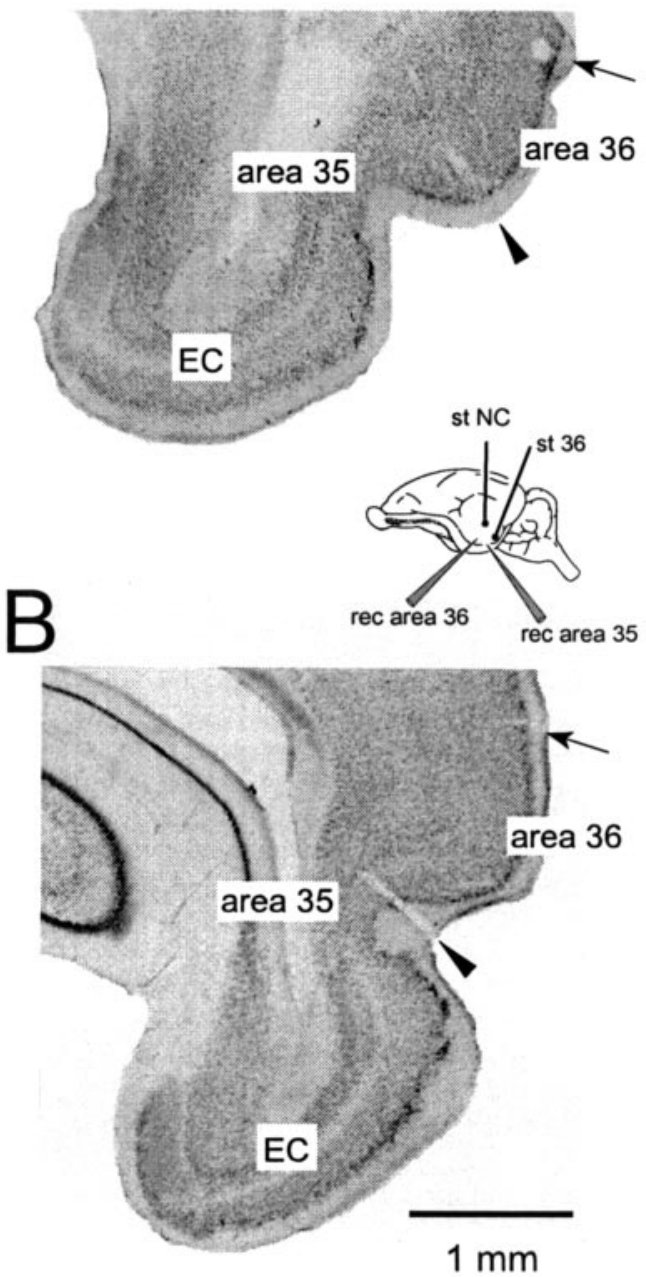

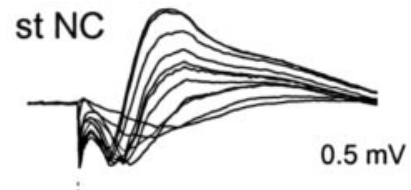
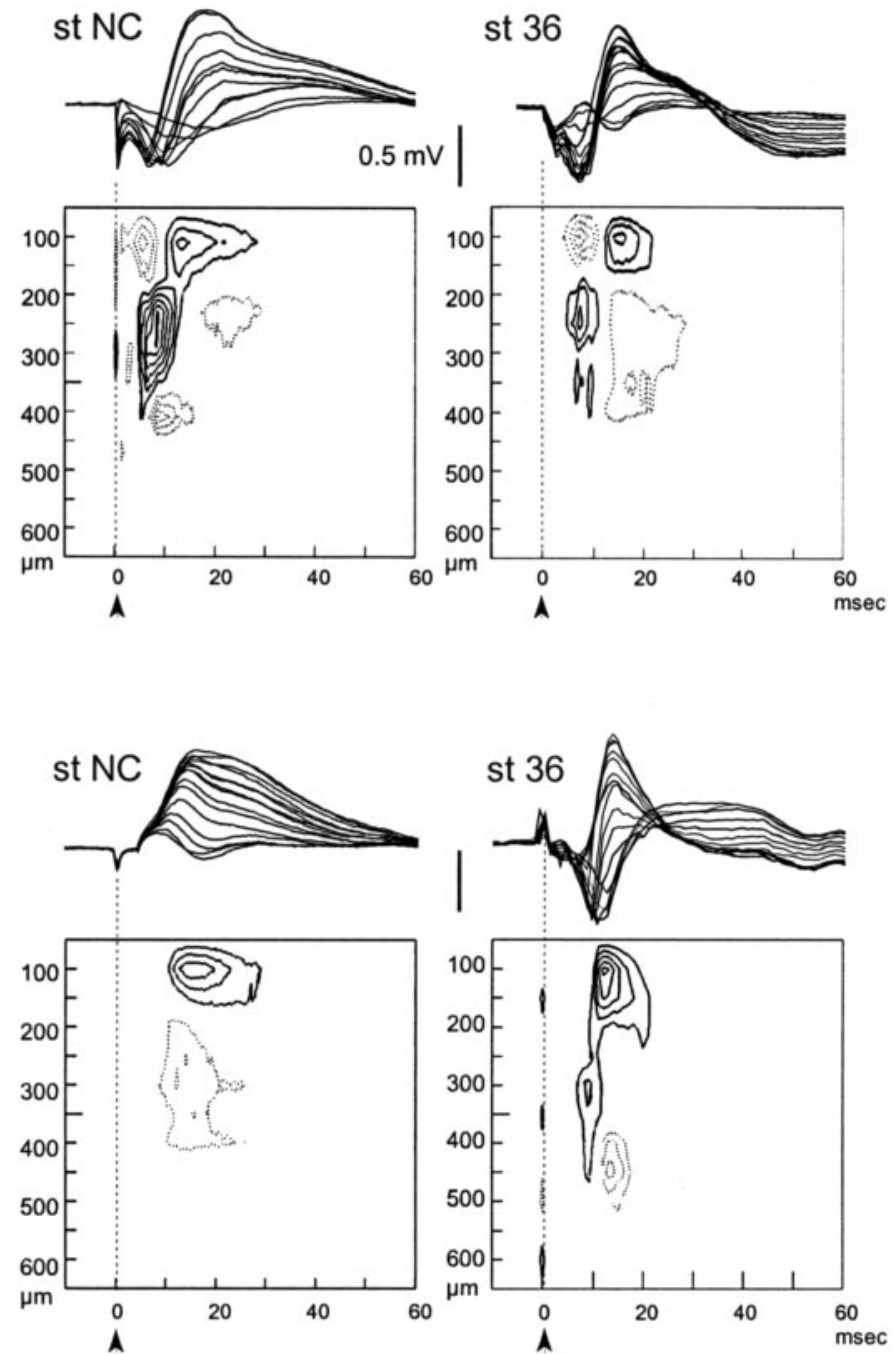

Figure 2. Typical field potential profiles and CSD contour plot recorded in area 36 in response to superficial stimulation of NC and area 36 . In $A$ and $B$, the correlation between the field potentials recorded with the multichannel silicon probe and the relative sink-source current dipoles plotted on a contour graph are shown. The field responses simultaneously recorded at each of the 16 probe sites (separated by $50 \mu \mathrm{m}$ on a single shaft orthogonally inserted in the cortex) are illustrated by superimposition in the top part of each panel. The continuous line and the dotted line in the contour plots indicate isocurrent lines referred to sinks and sources, respectively. Isocurrent lines are at $10 \mathrm{mV} / \mathrm{mm}^{2}$. The arrows in the contour plots mark the stimulus artifact. The recording silicon probes were inserted in area $36(B)$ and area $35(C)$. In the inset, a scheme of the position of the electrodes is illustrated. The histological controls in the left panels, illustrating the positions of the lesions performed by current passed through the stimulating electrode in the NC (arrow), and the lesion determined by the 16-channel silicon probe in area 36 (arrowhead) are illustrated in thionin-stained $100 \mu \mathrm{m}$ coronal sections.

in the EC (Fig. 4D, superimposed traces at the bottom). The amplitude and polarity of such potential did not vary with depth, suggesting that it represents a far field. CSD analysis of the same laminar profile did not show local sinks-sources, confirming that the potential is not generated locally (data not shown).

After NC stimulation, no responses could be evoked in the EC cells that regularly responded to LOT stimulation (data not shown). In 16 of 39 cells, stimuli in area 36 induced a late EPSP with onset latency of $17.65 \pm 9.1 \mathrm{msec}$ and peak amplitude delays $>25 \mathrm{msec}$ (Fig. $5 B$ ), whereas no response was recorded in the remaining 23 cells (Fig. $5 A$ ). Both groups of neurons were activated by LOT stimulation (Fig. 5, left traces in each panel). The study of the distribution of such neurons within the EC demonstrated that the large majority of neurons that responded with the late EPSP to area 36 stimulation was located in the m-EC (Fig. $5 B, G$, top panel).

In 11 of 32 EC neurons, area 35 stimulation induced EPSPs with variable onset, ranging from 13 to $35 \mathrm{msec}$ (average of $21.11 \pm 7.00 \mathrm{msec}$ ) and peak amplitude delays (between 15 and 50 msec) (Fig. $5 C-F$ ). Unlike area 36 stimulation, no preferential distribution of responsive neurons to either 1-EC or m-EC was found (Fig. 5G, bottom panel). The pure excitatory nature of the synaptic potentials evoked in EC by areas 35 and 36 stimulations has been demonstrated in seven cells by comparing traces obtained at different values of membrane potential (Fig. 6A,B). The EPSPs evoked by area 35 (middle traces) and area 36 stimulation (right traces) increased in duration when the membrane potential was artificially depolarized and eventually reached the threshold for generation of an action potential. No IPSPs were recorded in response to areas 35/36 stimulation. The presence of both fast and slow inhibitory responses that show typical membrane potential reversals of $\mathrm{GABA}_{\mathrm{A}}$ and $\mathrm{GABA}_{\mathrm{B}}$ receptor-mediated IPSPs was evoked in the same neurons by LOT stimulation (Fig. 6B, left panel). The latency of such IPSP is suggestive of a feedforward 

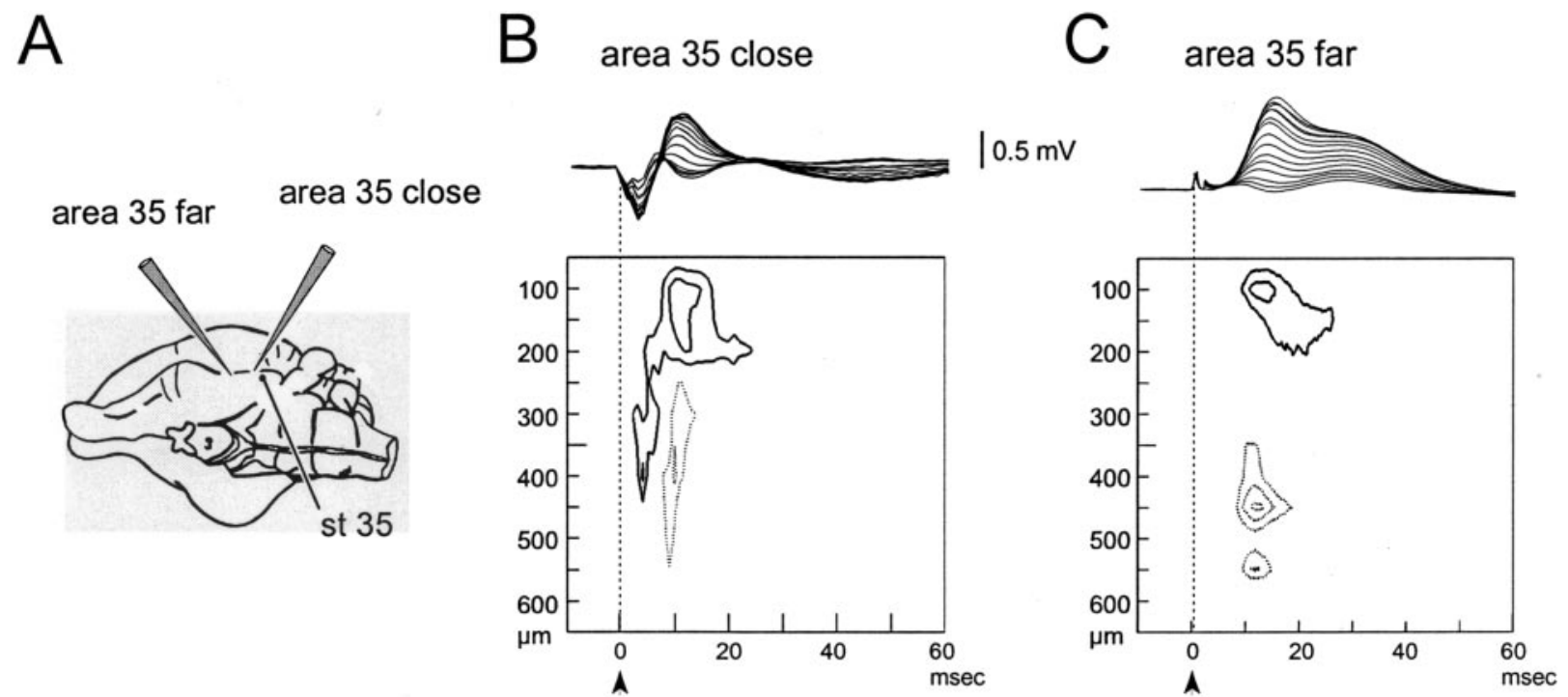

Figure 3. Field potential profiles and CSD contour plot recorded at two sites in area 35 located close to $(<1 \mathrm{~mm} ; B)$ and $2 \mathrm{~mm}$ away $(C)$ from a stimulating electrode in area 35 itself. The two profiles were performed in the same experiment. The positions of the electrodes are illustrated in $A$. As for Figure 2, the field profile and the CSD contour plot are illustrated in each panel. Isocurrent lines are at $10 \mathrm{mV} / \mathrm{mm}^{2}$.

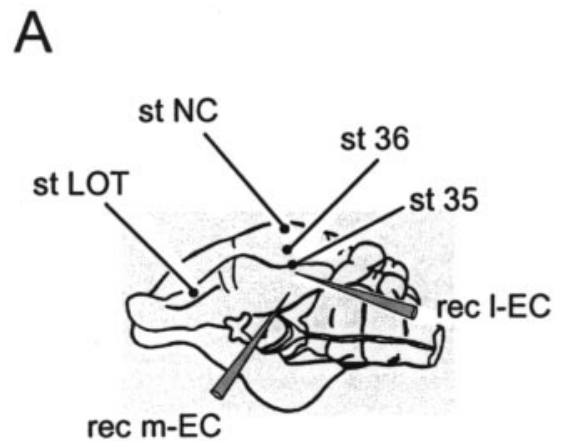

rec m-EC

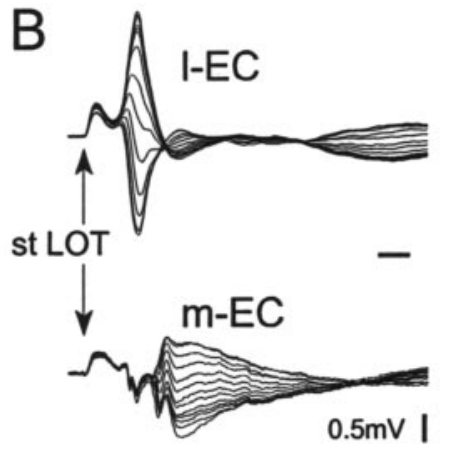

$0.5 \mathrm{mV}$ |
D

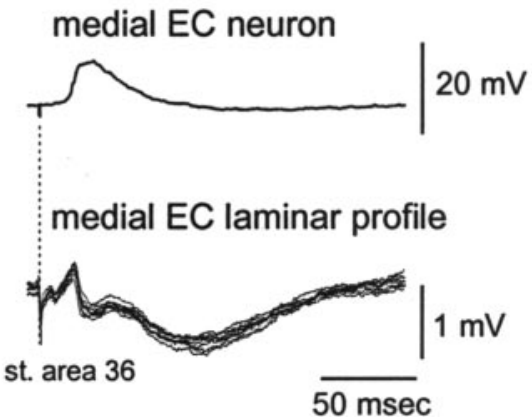

C

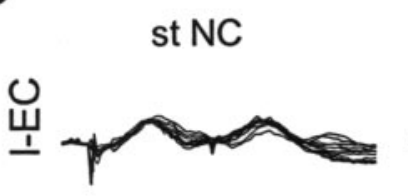

st 36
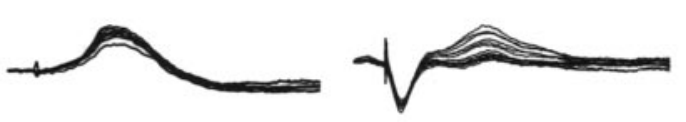

$0.2 \mathrm{mV}$
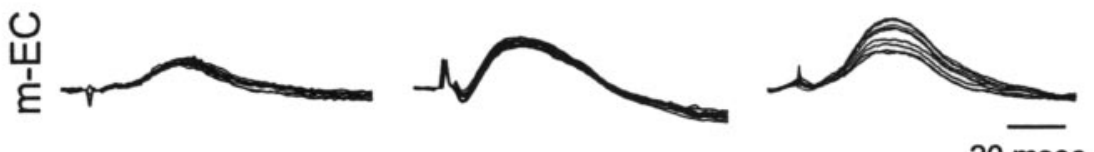

E

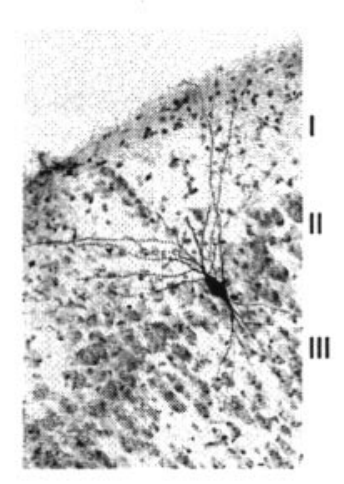

Figure 4. Field potential depth profiles recorded in m-EC and l-EC in response to stimulation of superficial NC, area 36, and area 35. A scheme of the position of the electrodes is illustrated in $A$. As illustrated in $B$, to guide the placement of the recording probes, we used the responses to LOT stimulation, characterized by potentials with different delays that showed a reversal within the cortical profile (Biella and de Curtis, 2000) in m-EC and 1-EC. Note that reversed potentials in l-EC have a shorter delay than in m-EC. $C$, Superimposed field responses to stimulation in NC, area 36, and area 35 recorded simultaneously with the 16 leads of a silicon probe inserted in the l-EC (top traces) and the m-EC (bottom traces). D, Simultaneous intracellular and field potential profile recorded in the medial EC after stimulation of superficial layer in area 36 . The intracellularly recorded neuron was filled with byocitin at the end of the electrophysiological recording and was identified as a layer II stellate neuron of the medial EC on the thionin-counterstained $79-\mu$ m-thick section illustrated in $E$. 


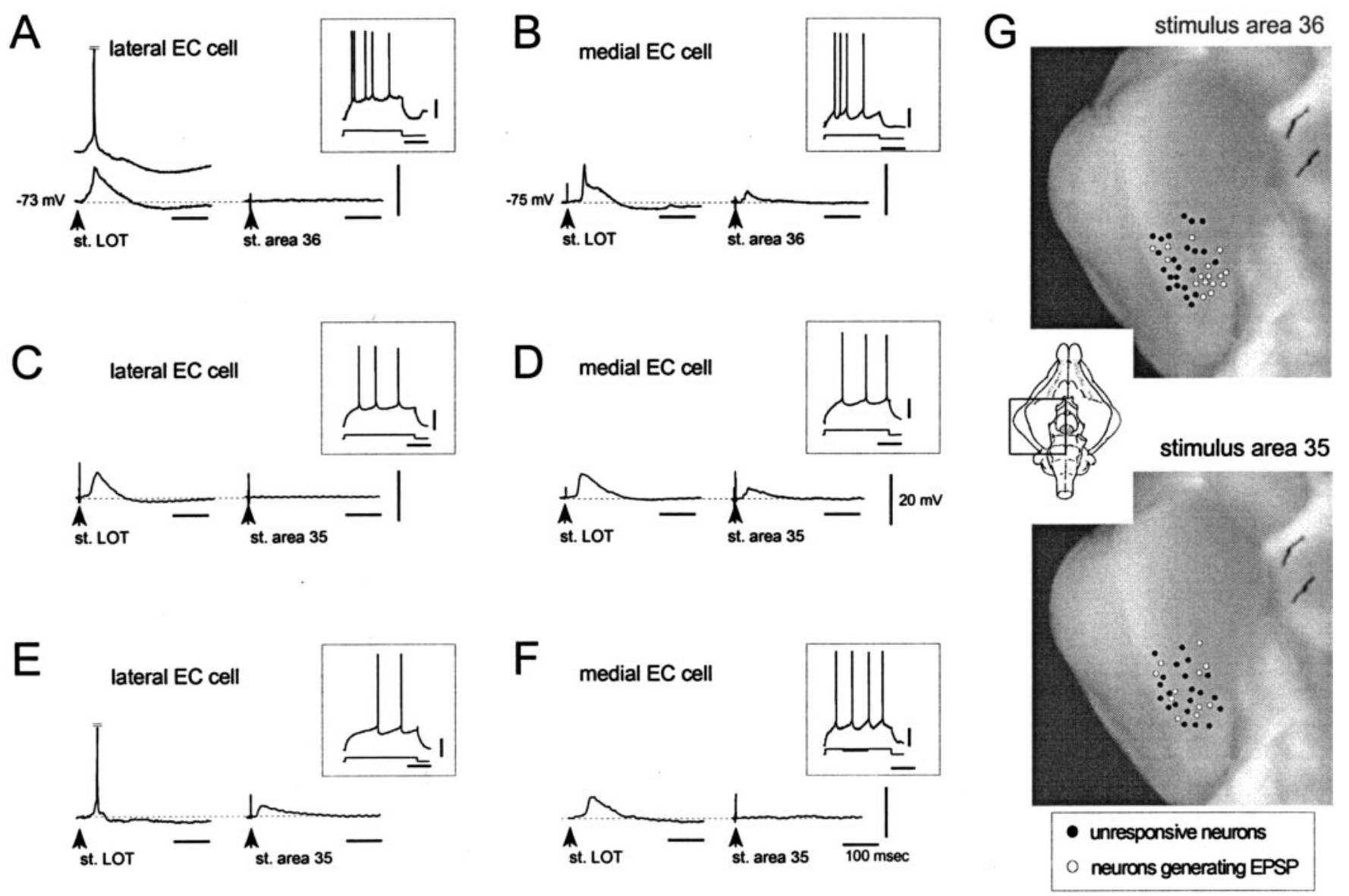

Figure 5. Typical activation patterns of EC layer II-III neurons recorded intracellularly in response to LOT (left traces) and PRC (right traces) stimulation. Neurons in $A, C$, and $E$ were recorded in the l-EC; neurons in $B, D$, and $F$ were recorded in m-EC. After area 36 stimulation, the large majority of l-EC cells did not respond $(A)$, whereas m-EC neurons generated an EPSP $(B)$. Area 35 stimulation induced EPSPs with variable delays in a subpopulation of l-EC and m-EC cells $(D, E)$. The same stimulus was ineffective in other EC neurons $(C, F)$. $G$, Distribution of EC neurons that generated an EPSP (open circles) and of cells unresponsive ( filled circles) to stimuli applied to area 36 (top panel) and area 35 (bottom panel). The large majority of neurons activated by area 36 stimulation were located in the m-EC and responded with a late, polysynaptic EPSP. The neurons that responded to area 35 stimulation with EPSPs of variable delays were scattered in both l-EC and m-EC.

inhibitory circuit (Finch et al., 1988; Funahashi and Stewart, 1998; Biella et al., 2001). No action potential firing was observed in EC neurons in response to LOT stimulation.

\section{DISCUSSION}

Anatomical findings support the view that the NC-to-EC associative pathway is formed by sequential step-like activation of longitudinal bands of cortex parallel to the rhinal sulcus. In the rat, Burwell and Amaral (1998a) demonstrated the existence of interconnections between area 36 and area 35 and between area 35 and subregions of the EC. Very weak connections were described between area 36 and the EC (Suzuki and Amaral, 1994; Naber et al., 1997; Burwell and Amaral, 1998b; Burwell, 2000). With the exception of a small contingent of deep layers neurons in the $\mathrm{NC}$ and the PRC that project to the PRC and the EC, respectively (McIntyre et al., 1996; Burwell and Amaral, 1998a,b), the largest contingent of fiber along the NC-PRC-EC pathway originate from layers II-III and project to the superficial layers of target areas (Deacon et al., 1983; Sorensen, 1985; Burwell et al., 1995; Burwell and Amaral, 1998a,b). Our findings demonstrate for the first time that a sequential cascade of excitatory synapses is activated in the parahippocampal cortices when the temporal $\mathrm{NC}$ is stimulated.
The study also suggests that the propagation of such activity is strongly regulated by an inhibitory control within the PRC.

\section{Neocortical input to the PRC and the EC}

Intracellular and extracellular recordings performed simultaneously in the whole isolated brain preparation (Biella et al., 2001) and in tangential PRC slices (Martina et al., 2001) of the guinea pig demonstrated that the local cortical circuit activated by temporal NC stimulation is characterized by a fast synaptic excitation spatially restricted in the rostrocaudal dimension, curtailed by a prominent feedforward inhibition and followed by an associative recurrent excitation that propagates longitudinally within the PRC, parallel to the rhinal sulcus. In the isolated guinea pig brain, the early monosynaptic EPSP recorded intracellularly was associated with an extracellular sink located at 200-400 $\mu \mathrm{m}$ depth, and both the disynaptic EPSP and IPSP were associated with a sink in the superficial $200 \mu \mathrm{m}$ (Fig. 2B). Superficial area 36 cells located at the periphery of the feedforward inhibitory influence generated an action potential in response to $\mathrm{NC}$ stimulation (Biella et al., 2001). The firing of these neurons is possibly responsible for the delayed, polysynaptic potential observed in area 35 layer I in response to NC stimulation. The absence of a 


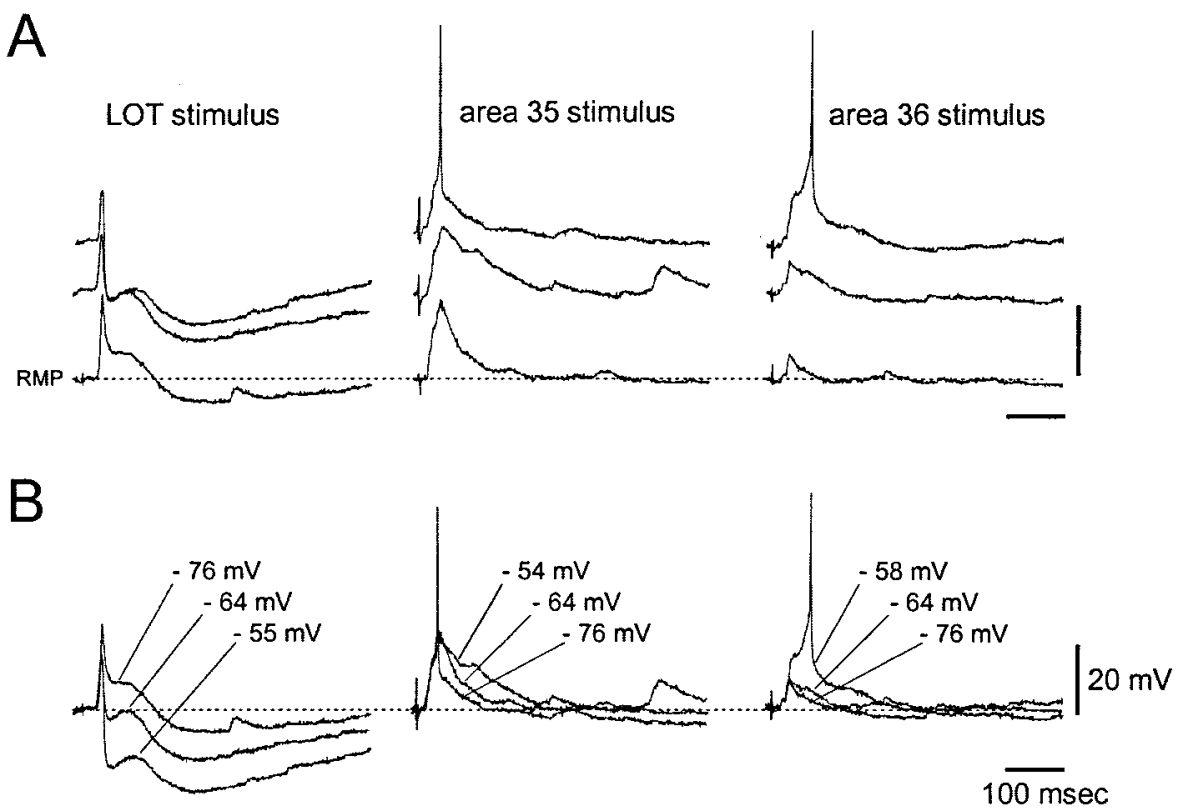

Figure 6. Intracellular recording performed at different membrane potentials in a single layer II neuron of the EC. Resting membrane potential was $-76 \mathrm{mV}$. Action potentials of $80 \mathrm{mV}$ amplitude were truncated. Responses to LOT stimulation, area 35 stimulation, and area 36 stimulation are illustrated in the left, middle, and right columns, respectively. Note that the early component of the LOT-induced IPSP is depolarizing at resting membrane potential. In $B$, the same responses were superimposed regardless of the real membrane potential values (indicated by the labeling) to show the changes of synaptic potential amplitude and polarity during membrane depolarization.

stimulation in our experiments was delivered in the most lateral part of area 36, which has been shown to project mainly within area 36 itself (Burwell and Amaral, 1998b). On the other hand, the diffuse EC distribution of fibers originating from area 36 described in the anatomical reports could be attributable to the anterograde transport of the tracer in area 35, which primarily projects to the lateral EC (Burwell and Amaral, 1998a).

Because EC and PRC are reciprocally connected, sparse synaptic activation of EC neurons in response to PRC stimulation could in principle occur via intracortical axon collaterals of antidromically activated cells. Such a possibility is unlikely for the following reasons: (1) no antidromic firing was ever observed in EC neurons in response to PRC stimulation, (2) lateral EC neurons (which, according to anatomical studies, project to the PRC) do not respond to area 36 stimulation, and (3) the onset latency of the polysynaptic EPSPs observed in EC cells is too long $(>15 \mathrm{msec})$ to be generated by antidromically activated EC cells.

Preliminary studies with voltage-sensitive dyes in combined disinhibited PRC-EC slices (M. P. Witter and T. Iijima, unpublished observations) showed that the propagation of neuronal activity from area 36 to the EC is mediated by a slowly developing, sustained activity in the deep layers at the border between the lateral EC and area 35. In addition, both anatomical studies and in vivo extracellular recordings demonstrated that area 36 projects to the subiculum (Naber and Witter, 1998), which could mediate an indirect activation of the EC (Naber et al., 1999). Therefore, the possibility that the area 36-evoked polysynaptic EPSPs in EC superficial layer cells could be mediated either via the activation of EC deep layers or the subiculum should be taken in consideration. Such a possibility should be verified with additional experiments.

\section{Area 35 input within area 35 and to the EC}

As for area 36, area 35 stimulation promotes associative propagation of synaptic potentials with a preferential longitudinal propagation, parallel to the rhinal fissure, within area 35 itself. Although a precise study of network interactions has not been possible in area 35 because of the difficulty to perform intracellular recordings in superficial layer neurons (see above), the CSD

pattern suggest that excitatory synaptic potentials in superficial could be attributable to the fact that, to avoid the possible costimulation of area 35 by diffusion of the current in the tissue, the anatomical data that demonstrated a diffuse, even if weak, projection from area 36 to the entire EC (Burwell and Amaral, 1998b), including the lateral band of cortex close to the sulcus in which no area 36-activated cells were found. This discrepancy 
layers sustain such intrinsic area 35 propagation. Surprisingly, stimulation of area 35 did not induce laminarly segregated local responses in the EC, because no definite current sinks were observed in EC profiles, although excitatory synaptic potentials could be evoked in a restricted number of layer II-III neurons recorded in both $\mathrm{m}-\mathrm{EC}$ and l-EC during intracellular recordings. The absence of a local extracellular potential in response to area 35 stimulation also suggests that area 35 neurons electroresponsiveness is dampened, possibly through inhibitory synaptic mechanisms similar to those described in area 36 (Biella et al., 2001; Martina et al., 2001). Besides the demonstration that such a connectivity is functionally "weak" and has a poor topographic organization, no clear regional segregation could be defined in the present study for the input from area 35 to the EC.

Although the organization of the functional interactions between parahippocampal subregions is still far from being defined, a new picture is emerging, according to which longitudinal bands of PRC and EC are efficiently interconnected (Biella et al., 2001, 2002; Martina et al., 2001), whereas the propagation of activity from the $\mathrm{NC}$ to the $\mathrm{EC}$ is under an efficient inhibitory control possibly generated within the PRC (Biella et al., 2001; Martina et al., 2001) that regulates the flow of information to the hippocampus. The results here described, indeed demonstrated, for the first time that the propagation of activity from area 36 and area 35 to the superficial layers of the EC is not massive and is spatially scattered. Such an organization contrasts with the amplitude of the potentials sustained by intrinsic associative connectivity within areas 36 and 35, as well as within EC (Biella et al., 2002). It is tempting to speculate that the strong associative connections within each rhinal subregion may play a determinant role in integrating inputs of neocortical origin that occur simultaneously, to generate neuronal firing, which could result in the propagation of excitation to the next synaptic station toward the hippocampus.

\section{REFERENCES}

Amaral DG (1999) What is where in the medial temporal lobe? Hippocampus 9:1-6.

Biella G, de Curtis M (1995) Associative synaptic potentials in the piriform cortex of the isolated guinea-pig brain in vitro. Eur J Neurosci 7:54-64.

Biella G, de Curtis M (2000) Olfactory inputs activate the medial entorhinal cortex via the hippocampus. J Neurophysiol 83:1924-1931.

Biella G, Uva L, de Curtis M (2000) Associative potentials in the pathway from the neocortex to the entorhinal cortex of the guinea-pig. Soc Neurosci Abstr 26:262.13.

Biella G, Uva L, de Curtis M (2001) Network activity evoked by neocortical stimulation in area 36 of the guinea pig perirhinal cortex. J Neurophysiol 86:164-172.

Biella G, Uva L, Hoffman U, de Curtis M (2002) Associative potentials in the entorhinal cortex of the guinea pig. J Neurophysiol 88:11591165.

Braak H, Braak E (1993) Entorhinal-hippocampal interaction in mnestic disorders. Hippocampus 3:239-246.

Brown MW, Aggleton JP (2001) Recognition memory: what are the roles of the perirhinal cortex and hippocampus? Nat Rev Neurosci 2:51-61.

Brown MW, Xiang JZ (1998) Recognition memory: neuronal substrates of the judgement of prior occurrence. Prog Neurobiol 55:149-189.

Burwell R (2000) The parahippocampal region: corticocortical interconnectivity. In: The parahippocampal region (Scharfman HE, Witter MP, Schwarcz R, eds), pp 25-42. New York: New York Academy of Sciences.

Burwell RD, Amaral DG (1998a) Perirhinal and postrhinal cortices of the rat: interconnectivity and connections with the entorhinal cortex. J Comp Neurol 391:293-321.

Burwell RD, Amaral DG (1998b) Cortical afferents of the perirhinal, postrhinal, and entorhinal cortices of the rat. J Comp Neurol 398:179-205.

Burwell RD, Witter MP, Amaral DG (1995) Perirhinal and postrhinal cortices of the rat: a review of the neuroanatomical literature and comparison with findings from the monkey brain. Hippocampus [Erratum (1996) 6:340] 5:390-408. de Curtis M, Pare D, Llinás RR (1991) The electrophysiology of the olfactory-hippocampal circuit in the isolated and perfused adult mammalian brain in vitro. Hippocampus 1:341-354.

de Curtis M, Biella G, Buccellati C, Folco G (1998) Simultaneous investigation of the neuronal and vascular compartments in the guinea pig brain isolated in vitro. Brain Res Brain Res Protoc 3:221-228.

Deacon TW, Eichenbaum H, Rosenberg P, Eckmann KW (1983) Afferent connections of the perirhinal cortex in the rat. J Comp Neurol 220:168-190.

Dolorfo CL, Amaral DG (1998) Entorhinal cortex of the rat: organization of intrinsic connections. J Comp Neurol 398:49-82.

Eichenbaum H, Otto T (1993) Odor-guided learning and memory in rats: is it "special"? Trends Neurosci 16:22-24.

Finch DM, Tan AM, Isokawa-Akeson M (1988) Feedforward inhibition of the rat entorhinal cortex and subicular complex. J Neurosci 8:2213-2226.

Funahashi M, Stewart M (1998) GABA receptor-mediated post-synaptic potentials in the retrohippocampal cortices: regional, laminar and cellular comparisons. Brain Res 787:19-33.

Insausti R, Herrero MT, Witter MP (1997) Entorhinal cortex of the rat: cytoarchitectonic subdivisions and the origin and distribution of cortical efferents. Hippocampus 7:146-183.

Ketchum KL, Haberly LB (1993) Membrane currents evoked by afferent fiber stimulation in rat piriform cortex. I. Current source-density analysis. J Neurophysiol 69:248-260.

Lopes da Silva FH, Witter MP, Boeijinga PH, Lohman AH (1990) Anatomic organization and physiology of the limbic cortex. Physiol Rev 70:453-511.

Martina M, Royer S, Paré D (2001) Propagation of neocortical inputs in the perirhinal cortex. J Neurosci 21:2878-2888.

McIntyre DC, Kelly ME, Staines WA (1996) Efferent projections of the anterior perirhinal cortex in the rat. J Comp Neurol 369:302-318.

Meunier M, Bachevalier J, Mishkin M, Murray EA (1993) Effects on visual recognition of combined and separate ablations of the entorhinal and perirhinal cortex in rhesus monkeys. J Neurosci 13:5418-5432.

Muhlethaler M, de Curtis M, Walton K, Llinás R (1993) The isolated and perfused brain of the guinea pig in vitro. Eur J Neurosci 5:915-926.

Naber PA, Witter MP (1998) Subicular efferents are organized mostly as parallel projections: a double-labeling, retrograde-tracing study in the rat. J Comp Neurol 393:284-297.

Naber PA, Caballero-Bleda M, Jorritsma-Byham B, Witter MP (1997) Parallel input to the hippocampal memory system through peri- and postrhinal cortices. NeuroReport 8:2617-2621.

Naber PA, Witter MP, Lopes da Silva FH (1999) Perirhinal cortex input to the hippocampus in the rat: evidence for parallel pathways, both direct and indirect. A combined physiological and anatomical study. Eur J Neurosci 11:4119-4133.

Otto T, Schottler F, Staubli U, Eichenbaum H, Lynch G (1991) Hippocampus and olfactory discrimination learning: effects of entorhinal cortex lesions on olfactory learning and memory in a successive-cue, go-no-go task. Behav Neurosci 105:111-119.

Sorensen KE (1985) Projection of the entorhinal area to the striatum, nucleus accumbens and cerebral cortex of the guinea pig. J Comp Neurol 238:308-322.

Steward O (1976) Topographic organization of the projections from the entorhinal area to the hippocampal formation of the rat. J Comp Neurol 167:285-314.

Suzuki WA (1996) The anatomy, physiology and functions of the perirhinal cortex. Curr Opin Neurobiol 6:179-186.

Suzuki WA, Amaral DG (1994) Topographic organization of the reciprocal connections between the monkey entorhinal cortex and the perirhinal and parahippocampal cortices. J Neurosci 14:1856-1877.

Swanson LW, Köhler C (1986) Anatomical evidence for direct projections from the entorhinal area to the entire cortical mantle in the rat. J Neurosci 6:3010-3023.

Uva L, Witter M, Wouterlood F, de Curtis M (2001) Cytoarchitectonic subdivisions and $3 \mathrm{D}$ reconstruction of the parahippocampal region of the guinea pig, Abstr D5. Proceedings Società Italiana Neuroscienze, Torino, Italy, September.

Van Hoesen G, Pandya DN (1975) Some connections of the entorhinal (area 28) and perirhinal (area 35) cortices of the rhesus monkey. I. Temporal lobe afferents. Brain Res 95:1-24.

Wilson RC, Steward O (1978) Polysynaptic activation of the dentate gyrus of the hippocampal formation: an olfactory input via the lateral entorhinal cortex. Exp Brain Res 33:523-534.

Witter MP (1993) Organization of the entorhinal-hippocampal system: a review of current anatomical data. Hippocampus 3:33-44.

Witter MP, Room P, Groenewegen HJ, Lohman AHM (1986) Connections of the PHC in the cat. V. Intrinsic connections; comments on input/output connections with the hippocampus. J Comp Neurol 252:78-94.

Zola-Morgan S, Squire LR, Amaral DG, Suzuki WA (1989) Lesions of perirhinal and parahippocampal cortex that spare the amygdala and hippocampal formation produce severe memory impairment. J Neurosci 9:4355-4370. 